\title{
A Canadian consensus forum on the management of patients with advanced prostate cancer
}

Fred Saad $^{1}$; Christina Canil ${ }^{2}$; Antonio Finelli ${ }^{3}$; Sebastien J. Hotte ${ }^{4}$; Shawn Malone ${ }^{2}$; Bobby Shayegan $^{5}$; Alan I. So ${ }^{6}$; Lorne Aaron ${ }^{7}$; Naveen S Basappa ${ }^{8}$; Henry J Conter ${ }^{9}$; Brita Danielson ${ }^{8}$; Geoffrey Gotto $^{10}$; Robert J. Hamilton ${ }^{3}$. Jason P. Izard ${ }^{11}$; Anil Kapoor ${ }^{5}$; Michael Kolinsky ${ }^{8}$; AlyKhan A. Lalani ${ }^{4}$; Jean-Baptiste Lattouf ${ }^{1}$; Christopher Morash ${ }^{2}$; Scott C. Morgan ${ }^{2}$; Tamim Niazi ${ }^{12}$; Krista L. Noonan ${ }^{13}$; Michael Ong ${ }^{2}$; Ricardo A. Rendon ${ }^{14}$; Sandeep Sehdev ${ }^{2}$; Huong Hew ${ }^{15}$; Laura Park-Wyllie ${ }^{15}$; Kim N Chi ${ }^{13}$

${ }^{1}$ Centre Hospitalier de l'Université de Montréal, University of Montreal, Montreal, QC, Canada; ${ }^{2}$ The Ottawa Hospital, University of Ottawa, Ottawa, ON, Canada; ${ }^{3}$ Princess Margaret Cancer Centre, University of Toronto, Toronto, ON, Canada; ${ }^{4}$ Juravinski Cancer Centre, McMaster University, Hamilton, ON, Canada; ${ }^{5}$ St. Joseph's Healthcare, McMaster University, Hamilton, ON, Canada; ${ }^{6}$ Prostate Centre at Vancouver General Hospital, University of British Columbia, Vancouver, BC, Canada; ${ }^{7}$ Service d-Urologie and Centre de la Prostate, Longueuil, QC, Canada ; ${ }^{8}$ Cross Cancer Institute, University of Alberta, Edmonton, AB, Canada; ${ }^{9}$ William Osler Health System, University of Western Ontario, Brampton, ON, Canada; ${ }^{10}$ Southern Alberta Institute of Urology, University of Calgary, Calgary, AB, Canada; ${ }^{11}$ Kingston Health Sciences Centre, Queen's University, Kingston, ON, Canada; ${ }^{12}$ Jewish General Hospital, McGill University, Montreal, QC, Canada; ${ }^{13}$ BC Cancer Agency, University of British Columbia, Vancouver, BC, Canada; ${ }^{14}$ Queen Elizabeth II Health Sciences Centre, Dalhousie University, Halifax, NS, Canada; ${ }^{15}$ Medical Affairs, Janssen Inc, Toronto, ON, Canada

Acknowledgements: The authors would like to thank Dr. Neil Fleshner, Dr. Frederic Pouliot, and Dr. Jeffrey Spodek for their contributions to the development of the consensus questions. They would also like to thank Delna Sorabji and her contributions to the design and execution of the project, as well as Timothy Doyle and his work providing editorial assistance.

Cite as: Can Urol Assoc J 2019 November 5; Epub ahead of print. http://dx.doi.org/10.5489/cuaj.6082

Published online November 5, 2019

$* * *$

\section{Abstract}

Introduction: The management of advanced prostate cancer $(\mathrm{PCa})$ continues to evolve with the emergence of new diagnostic and therapeutic strategies. As a result, there are multiple areas in this landscape with a lack of high-level evidence to guide practice. Consensus initiatives are an approach to establishing practice guidance in areas where evidence is unclear. We conducted a Canadian-based consensus forum to address key controversial areas in the management of advanced PCa. 
Methods: As part of a modified Delphi process, a core scientific group of PCa physicians $(\mathrm{n}=8)$ identified controversial areas for discussion and developed an initial set of questions, which were then reviewed and finalized with a larger group of 29 multidisciplinary PCa specialists. The main areas of focus were non-metastatic castration-resistant prostate cancer (nmCRPC), metastatic castration-sensitive prostate cancer (mCSPC), metastatic castration-resistant prostate cancer (mCRPC), oligometastatic prostate cancer, genetic testing in prostate cancer, and imaging in advanced prostate cancer. The predetermined threshold for consensus was set at $74 \%$ (agreement from 20 of 27 participating physicians).

Results: Consensus participants included uro-oncologists $(n=13)$, medical oncologists $(n=10)$, and radiation oncologists $(n=4)$. Of the 64 questions, consensus was reached in 30 questions $(n=5$ unanimously). Consensus was more common for questions related to biochemical recurrence, sequencing of therapies, and mCRPC.

Conclusions: A Canadian consensus forum in PCa identified areas of agreement in nearly 50\% of questions discussed. Areas of variability may represent opportunities for further research, education, and sharing of best practices. These findings reinforce the value of multidisciplinary consensus initiatives to optimize patient care.

\section{Introduction}

Prostate cancer (PC) is one of the leading causes of cancer-mortality in men. ${ }^{1}$ Despite recent advances in the diagnostic and therapeutic strategies for management of prostate cancer, there are still multiple areas in this landscape deficient of high-level evidence to guide practice. A multidisciplinary consensus approach can provide guidance in areas where evidence is lacking or unclear. ${ }^{2}$ A Canadian-based consensus forum to address key controversial areas in the management of advanced prostate cancer was conducted.

\section{Methods}

We used a modified Delphi process for the Genitourinary Research Consortium (GURC) Canadian Consensus Forum (referred to as the Consensus Forum from here on in) as shown in Figure 1 and modelled after other global consensus initiatives. The GURC is a multidisciplinary scientific group of prostate cancer physicians across Canada who collaborate on scientific, educational, and best practice initiatives. ${ }^{2}$ First, a core scientific group of 8 multidisciplinary prostate cancer expert physician (GU Research Consortium (GURC) Steering Committee) identified the key areas of focus for the consensus forum, and then drafted the initial set of consensus questions. After completing more than 2 rounds of review, questions were refined, and the number of questions were reduced.

Second, a panel of 29 Canadian multidisciplinary prostate cancer specialists were invited to review and provide feedback on the draft questions. Panel participants consisted of all members 
of GURC Steering Committee, Scientific Task Force, Education, and Best Practice Working Groups. The panel reviewed and refined each question and set of responses.

At the completion of the review period, 64 questions were prioritized for the Consensus Forum day and 50 questions delegated to the supplemental online consensus process. The panel was invited to participate in the pre-testing of the consensus questions.

On the day of the Consensus Forum, the core scientific group led the panel of 27 clinicians in discussion, debate, and clarification of questions before anonymous voting. Two members of the 29-member GURC were unable to join the consensus voting forum day. The predetermined threshold for consensus was set at 74\% (agreement from 20 of 27 participating physicians) similar to other consensus initiatives. ${ }^{2-4}$ In deliberating their answers, participants agreed no barriers to treatment access would be assumed. Where appropriate, the panel determined if there was a need to repeat the vote on a question following clarification or refinement of the question. ${ }^{5-7}$

The supplemental questions underwent consensus voting during the week following the Forum day via an online survey.

\section{Results}

The main areas of focus selected for the Forum were as follows:

1. Biochemical recurrence following local definitive therapy

2. Non-metastatic castration-resistant prostate cancer (nmCRPC)

3. Metastatic castration-sensitive prostate cancer (mCSPC)

4. Sequencing of systemic treatments

5. Metastatic castration-resistant prostate cancer (mCRPC)

6. Oligometastatic prostate cancer

7. Access and funding of treatments

8. Genetic testing in prostate cancer

9. Referrals for care

10. Imaging in advanced prostate cancer

The consensus forum was comprised of urologists $(n=13,48 \%)$, medical oncologists $(n=10$, $37 \%)$ and radiation oncologists $(n=4,15 \%)$. Geographic representation spanned British Columbia and Alberta $(n=7,26 \%)$, Ontario $(n=15,56 \%)$, and Quebec and Atlantic Canada $(n=5,19 \%)$. The majority of panel participants had been in practice for greater than 10 years $(n=17,64 \%)$.

In general, of the 64 questions that underwent anonymous in-person electronic voting at the Forum, consensus was reached in 30, with unanimous voting in 5 questions. (Table 1) Group moderated discussions followed the voting for each question. Eight questions underwent re-voting after either clarifying discussion or question refinements. Question 7 was eliminated after it was deemed no longer relevant after question revision. One question was added during the consensus forum (Q22b). Consensus was more common prevalent for questions related to biochemical recurrence, sequencing of therapies, and $\mathrm{mCRPC}$ and less common for oligometastatic prostate 
cancer, metastatic castration-sensitive prostate cancer, and non-metastatic castration-resistant prostate cancer. The voting results for each question are available in supplementary Appendix 2.

Of the supplementary questions that occurred via an online survey following Consensus Forum, threshold of consensus (>74\%) was reached in 19 of 50 questions.

\section{Reporting areas of consensus, simple majority, and variability}

Areas of consensus and related discussion are reported below. Selected areas of simple majority vote ( $>50 \%$ agreement), and areas of variability are reported below and in the Supplemental Appendix with complete voting results.

\section{Biochemical recurrence following radical therapy}

Consensus was reached for the following questions: when to begin ADT for biochemical recurrence following local definitive therapy, what type of ADT to use, and the frequency of PSA monitoring while on ADT for biochemical recurrence following definitive local therapy.

When to begin ADT: $89 \%$ of physicians indicated that they primarily look at absolute PSA level (majority use $>5 \mathrm{ng} / \mathrm{mL}$ post radical prostatectomy or $>10 \mathrm{ng} / \mathrm{mL}$ post radical radiation therapy) to determine when to begin ADT for PSA recurrence following local radical therapy. Other factors that did not reach consensus were PSADT $\leq 10$ months $(63 \%$ of physicians) and high-risk pathologic features (i.e. high Gleason score) (33\% of physicians).

What method of ADT: $93 \%$ of physicians recommend initiating intermittent ADT for PSA-only recurrence following local radical therapy rather than continuous ADT.

Frequency of PSA monitoring while on ADT for biochemical recurrence: $93 \%$ of physicians indicated monitoring PSA every 3-4 months during ADT treatment for PSA recurrence. The panel commented that this frequency of testing reflects a need for timely assessment of PSA doubling time in patients developing castration resistance.

\section{Non-metastatic castrate-resistant prostate cancer}

Consensus was reached for the PSADT threshold on which to begin treatment for nmCRPC, interpretation of PSADT in the context of imaging results, and use of a metastasis-free survival (MFS) endpoint to determine start of second-generation androgen receptor (AR) targeted therapy (ARAT).

PSADT threshold to begin treatment in nmCRPC: $78 \%$ of physicians indicated using a PSADT threshold of $\leq 10$ months to begin 2nd-generation ARAT in nmCRPC. During the discussion, some participants discussed that the PSADT in the SPARTAN ${ }^{8}$ and PROSPER $^{9}$ study population was $<6$ months for the majority of study patients. However, participants also discussed that all patients with PSADT $\leq 10$ months (both $<6$ months and $>6$ months) were observed to benefit from therapy. 
Role of absolute PSA levels: Although there is evidence for elevated absolute PSA levels as a predictive marker of time to metastases and overall survival ${ }^{10-11}$, when the panel was asked to consider a patient with elevated PSA between 10-20 ng/mL and PSADT greater than 10 months, the panel consensus ( $89 \%$ of physicians) was that they would still use PSADT $\leq 10$ months as a trigger to begin treatment. In patients with absolute PSA of $20-40 \mathrm{ng} / \mathrm{mL}, 30 \%(\mathrm{n}=8)$ of physicians considered initiating second-generation AR therapy, despite PSADT of $>10$ months.

PSADT in the context of imaging results: When PSADT $\leq 10$ months and conventional imaging is negative for metastases, $96 \%$ of physicians indicated they would treat with agents approved for nmCRPC such as apalutamide or enzalutamide. Since the Consensus Forum occurred prior to the availability of evidence for darolutamide, darolutamide could not be included in the consensus questions, however the Phase 3 data has since been published and a similar indication is anticipated from Health Canada. When PSADT $\leq 10$ months, conventional imaging is negative for metastases, and PET-based imaging is positive for metastases, $89 \%$ of physicians indicated they would treat these patients with nmCRPC agents such as apalutamide or enzalutamide. Regarding this latter scenario, the panel acknowledged the classifying patients as simply "nonmetastatic" or "metastatic" is complicated in the context of advanced imaging modalities, and beyond the scope of the questions posed at the Consensus Forum. However, the panel concluded that, regardless of the classification, patients with no metastases on conventional imaging and a PSADT $\leq 10$ months, are indeed the patients studied in the SPARTAN ${ }^{8}$ and PROSPER $^{9}$ studies and thus are the patients expected to derive the benefits reported in these studies. On balance, the panel concluded that the available evidence supports treatment of these patients with apalutamide or enzalutamide regardless of PET imaging.

MFS endpoint for treatment decision-making in nmCRPC

The use of the MFS endpoint was found to be sufficient for treatment decision-making in nmCRPC. Physicians were in full agreement (100\% consensus) that surrogate endpoints likely correlated with OS such as MFS, provide sufficient evidence for treatment decision-making in nmCRPC. During the discussion, it was highlighted that the positive voting results of this question reflected the large magnitude of MFS benefit reported by the $\operatorname{SPARTAN}^{8}$ and PROSPER $^{9}$ studies.

\section{Metastatic castration-sensitive prostate cancer}

Consensus was reached in the type of ADT used for high-volume mCSPC and the treatment of the primary in low volume/low risk patients and high-volume/high risk patients.

Type of ADT used for high-volume mCSPC: $81 \%$ of physicians use continuous LHRH agonist $+/$ - short course first-generation anti-androgen in the majority of their patients, $11 \%$ use LHRH antagonists and 7.4\% use continuous complete androgen blockage (LHRH analog +/ firstgeneration $\mathrm{AR}$ antagonists) 
Treatment of primary: In de novo low-volume asymptomatic mCSPC, $74 \%$ of physicians indicated they would treat the primary in addition to systemic therapy while $19 \%$ of physicians indicated they would consider treatment to the primary in select patients. In terms of the preferred type of treatment, $96 \%$ of physicians indicated that radiation therapy was preferred based on recent evidence from the STAMPEDE trial. ${ }^{12}$ In de novo high-volume asymptomatic mCSPC, $78 \%$ of physicians indicated they would not recommend treatment of primary in addition to systemic therapy, but during the discussion, the panel acknowledged that radiation is sometimes still given to patients in whom there are concerns about local symptoms and local progression and may be on the borderline of the low volume definition.

\section{Sequencing of treatments}

Consensus was reached across all treatment sequencing scenarios including nmCRPC to mCRPC, mCSPC to $\mathrm{mCRPC}$, and mCRPC first-line to second-line therapy.

nmCRPC to mCRPC: If apalatumide or enzalutamide was for nmCRPC, $>80 \%$ of physicians indicated they would sequence to docetaxel.

mCSPC (docetaxel) to mCRPC: If docetaxel was used for mCSPC followed by asymptomatic or minimally symptomatic progression, $100 \%$ of physicians indicated they would sequence to abiraterone plus prednisone, or enzalutamide for mCRPC. If the patient was symptomatic, $70 \%$ of physicians indicated they would sequence to abiraterone plus prednisone or enzalutamide, $15 \%$ of physicians indicated they would use docetaxel for mCRPC, followed by $\sim 7.5 \%$ who would use cabazitaxel and $\sim 7.5 \%$ who would use Radium-223. This scenario assumed that the patient had received and responded to 6 cycles of docetaxel and were now progressing symptomatically with no visceral metastases. A few panel members commented that the evidence for docetaxel re-challenge was limited and if the disease-free interval was short between completion of docetaxel and progression of symptoms, the patient would be unlikely to respond to docetaxel re-challenge.

mCSPC (abiraterone plus prednisone) to mCRPC: If abiraterone plus prednisone was used for mCSPC and the patient was asymptomatic or minimally symptomatic, $78 \%$ of physicians indicated they would sequence to docetaxel, and $15 \%$ of physicians indicated they would sequence to enzalutamide for mCRPC. During the discussion, some physicians explained their preference for use of enzalutamide after abiraterone plus prednisone in this setting by citing the Chi Crossover study ${ }^{13}$ and the opportunity to avoid or delay chemotherapy in mCRPC population. If abiraterone plus prednisone was used for mCSPC and the patient was symptomatic, $96 \%$ of physicians indicated they would sequence to docetaxel for $\mathrm{mCRPC}$.

mCRPC $1^{\text {st }}$ line (ARAT) to $2^{\text {nd }}$ line: If the first-line treatment was abiraterone plus prednisone or enzalutamide and the patient was experiencing asymptomatic PSA progression, $78 \%$ of physicians indicated they would continue the current treatment course. However, if the patient was exhibiting asymptomatic radiographic + PSA progression, 100\% of physicians indicated they would switch to docetaxel. If progression was symptomatic progression, $96 \%$ of 
physicians indicated they would switch to docetaxel for $2^{\text {nd }}$ line treatment. Given the limited data for radium-223, the Consensus Forum did not consider scenarios where radium-223 was initiated for first line mCRPC.

mCRPC $1^{\text {st }}$ line (docetaxel) to $2^{\text {nd }}$ line: If the first-line treatment was docetaxel and there was no prior use of abiraterone plus prednisone or enzalutamide, $100 \%$ of physicians indicated they would use abiraterone plus prednisone or enzalutamide as $2^{\text {nd }}$ line treatment.

Chemotherapy sandwiched between ARAT sequencing: The panel did not feel that chemotherapy used after initial ARAT therapy restores sensitivity to further ARAT use (74\% of physicians). During the discussion, the panel discussed that while some data exists that suggests a better response when chemotherapy is used between AR therapy, it is more likely that this is related to a break from AR therapy, which allows for re-proliferation of hormone-sensitive clones before re-exposure. ${ }^{13-16}$

Sequencing combinations for $\mathrm{nmCRPC}$ to $\mathrm{mCRPC}$ and $\mathrm{mCSPC}$ to $\mathrm{mCRPC}$ are shown in Figure 2.

\section{Metastatic castration-resistant prostate cancer}

Consensus was reached for the selection of first-line treatment and steroid-switching for rising PSA among patients taking abiraterone plus prednisone.

First-line treatment CRPC: For men who were not previously treated with docetaxel or abiraterone plus prednisone in the mCSPC setting, the vote was unanimous as $100 \%$ of physicians indicated they would use abiraterone plus prednisone or enzalutamide.

Steroid switch: $85 \%$ of physicians indicated they would switch from prednisone to dexamethasone in patients treated with abiraterone plus prednisone for mCRPC who exhibited PSA progression alone. During the discussion, the panel also elaborated that referral to medical oncologist should be considered at the time of steroid switch and restaging should be ordered.

\section{Oligometastatic prostate cancer}

No questions reached the threshold level of consensus in this section. Greater than $50 \%$ of physicians agreed on a response for several questions but not at the level of consensus.

\section{Access to treatments}

No questions reached the threshold level of consensus.

\section{Genetic testing in prostate cancer}

In patients with DNA repair defects (germline or somatic) who progress early on ADT, 78\% of physicians (consensus) indicated they would treat with standard first-line treatment for mCRPC.

Newly diagnosed metastatic prostate cancer: Panel consensus (74\%) was reached that genetic counselling and testing should be conducted in select patients with newly diagnosed metastatic prostate cancer. Further discussion of which select patients were most appropriate for genetic counselling and testing revealed that $89 \%$ of physicians would recommend testing for 
men with positive family history for prostate, breast, and ovarian cancer. Other circumstances for testing were patients with positive family history of other cancers ( $75 \%$ of physicians), younger men $<60$ years of age at diagnosis $(67 \%)$ of physicians, patients with visceral metastases $(48 \%$ of physicians), and intraductal or cribriform pathology (44\% of physicians).

Approximately one-quarter of physicians (26\%) indicated they would recommend genetic counselling and testing in the majority of patients.

\section{Referrals}

Consensus agreement was not reached on the referral-based questions.

\section{Imaging}

PET-CT imaging to diagnose oligometastatic recurrence: Consensus was reached in the role of PET-CT imaging to diagnose oligometastatic recurrent state in men with CSPC after local treatment with curative intent (+/- salvage radiation therapy), where $74 \%$ of physicians recommend PET-CT in this scenario and 26\% recommend standard CT and/or MRI and bone scintigraphy.

CT and bone scintigraphy for mCSPC: Consensus was also reached in the use of CT and bone scintigraphy for majority of men with $\mathrm{mCSPC}$, where $78 \%$ would use standard $\mathrm{CT}$ and bone scintigraphy, and $22 \%$ would use next-generation imaging.

\section{Discussion}

Physicians treating advanced prostate cancer face challenges of making treatment decisions in the face of a paucity of high-level evidence to guide practice. Consensus development methods may be useful in these types of situations by providing another mechanism to synthesize available evidence together with expert opinion and contextual patient factors to develop recommendations.

We applied a modified Delphi process to engage the GURC panel in several rounds of review and feedback to develop and refine a comprehensive survey of practice questions. This was followed by a consensus development day that enabled the GURC panel to meet in-person to discuss and vote on each practice question, and ultimately identify areas of consensus using a predefined threshold of $74 \%$ agreement. As a panel, we identified 30 consensus areas in advanced prostate cancer management.

Consensus aligned with existing high-quality evidence when available, and also reflected rapid uptake and synthesis of new evidence in emerging areas such as nmCRPC and mCSPC. In nmCRPC, physicians were in consensus agreement to treat their castration-resistant patients who were negative for metastases on conventional imaging with apalatumide or enzalutmide with ongoing ADT when PSADT dropped to $<10$ months, aligning to the recent data from the SPARTAN8 and PROSPER9 studies. In MCSPC, there was consensus agreement for treating the primary tumour with radiation therapy in patients with low-volume prostate cancer which aligns with recent data from the STAMPEDE radiotherapy analysis. ${ }^{12}$ 
Consensus was also observed in situations where level 1 evidence does not exist. In particular, a high degree of consensus was seen in treatment sequencing practices despite the limited data available to guide these types of treatment decisions.

Establishing consensus for a sequencing approach for treatments across the disease spectrum was a key output of the expert panel. After use of apalutamide or enzalutamide with ongoing ADT for nmCRPC, the consensus of the panel was to sequence to docetaxel upon progression to $\mathrm{mCRPC}$. In $\mathrm{mCSPC}$, if progression occurred following docetaxel treatment, the consensus was to sequence next to abiraterone acetate + prednisone, or enzalutamide for mCRPC. Similarly, if progression occurred while on abiraterone acetate plus prednisone, then the panel was in consensus to sequence to docetaxel therapy for mCRPC. Lastly, for mCRPC, if patients had not been previously treated with next generation AR therapy or docetaxel, the panel was unanimous that they would use abiraterone acetate plus prednisone or enzalutamide for first-line mCRPC followed by docetaxel for second line therapy. If docetaxel had been used in first-line mCRPC, they were in full consensus for using abiraterone acetate plus prednisone, or enzalutamide for second-line treatment.

Over the course of the consensus discussions, the panel identified several areas where additional research is critically needed to guide more informed treatment decisions.

Oligometastatic disease, in particular, was felt to require substantially more research, starting with a unified definition of what represents clinically meaningful oligometastatic disease, the role of stereotactic body radiation therapy as metastasis-directed therapy for oligometastatic castrationresistant and castration-sensitive disease, treatment of newly-diagnosed castration-sensitive oligometastatic disease and oligometastatic recurrent castration-sensitive disease.

The role of genetic testing was also flagged as an area for further research, especially how the presence of DNA repair defects could influence treatment such as use of PARP inhibitors and treatment decisions in $\mathrm{MCRPC}$, and the role of genetic testing in newly diagnosed metastatic cancer and localized prostate cancer.

There are some limitations associated with this work. First, the evidence in this field is changing rapidly, and while this report provides a strong snapshot of current practice in Canada from a highly-specialized multidisciplinary panel, it is at risk of becoming outdated quickly as more evidence emerges. The consensus voting occurred in December 2018 and was based on the available data at the time. The evidence for newer agents such as darolutamide became available after the consensus forum. Second, the consensus panel was primarily composed of academic hospital-based specialists, some types of specialists such as radiation oncologists were underrepresented on the consensus panel, and future research is needed to understand the extent to which practice in the community-setting is similar or different from the specialist panel. Third, because many of the recommendations are based on expert opinion, it is important to note that the 
information used to formulate the recommendations is of a lower level of evidence relative to randomized trials and other forms of interventional research.

Despite these limitations, we feel that the consensus recommendations arising from the Consensus Forum addresses several important gaps in the management of advanced prostate cancer and will be of value to Canadian prostate cancer physicians. The consensus development process was able to capture how leading Canadian prostate cancer physicians have synthesized the best available data and then incorporated other key knowledge of patient risk factors, treatment history, and drug characteristics in order to guide treatment decision-making in the absence of level 1 evidence. The consensus recommendations are also the product of a diverse scientific panel of multidisciplinary experts and represent important areas of Canadian consensus, and in some cases, lack of consensus across Canada.

\section{Conclusions}

A Canadian Consensus Forum of prostate cancer specialists identified areas of consensus in nearly $50 \%$ of questions discussed. The remaining questions showed a range simple majority agreement or considerable variability in practice across the remaining questions. These results reflect real-world practice. Areas of variability may represent opportunities for further research, education, and sharing of best practices. These findings reinforce the value of multidisciplinary consensus initiatives to optimize patient care. 


\section{References}

1. Cronin KA, Lake AJ, Scott S, et al. Annual report to the nation on the status of cancer, part I: national cancer statistics. Cancer 2018;124:2785-800.

2. Gillessen S, Attard G, Beer TM, et al. Management of patients with advanced prostate cancer: The report of the advanced prostate cancer consensus conference APCCC 2017. Eur Urol 2018;73:178-211. Epub 2017 June 29.

3. Morgans AK, van Bommel AC, Stowell C, et al. Development of a standardized set of patient-centered outcomes for advanced prostate cancer: an international effort for a unified approach. Eur Urol 2015;68:891-8. Epub 2015 July 2.

4. Diamond IR, Grant RC, Feldman BM, et al. Defining consensus: a systematic review recommends methodologic criteria for reporting of Delphi studies. J Clin Epidemiol 2014;67:401-9. Epub 2014 March 4.

5. Jones J, Hunter D. Consensus methods for medical and health services research. BMJ 1995;311:376-80.

6. O'Donovan A, Mohile SG, Leech M. Expert consensus panel guidelines on geriatric assessment in oncology. Eur J Cancer Care 2015;24(4):574-89.

7. Kea B, Sun BC. Consensus development for healthcare professionals. Intern Emerg Med 2015;10(3):373-83.

8. Smith MR, Saad F, Chowdhury S, et al. Apalutamide treatment and metastasis-free survival in prostate cancer. New Engl J Med 2018;378:1408-18. Epub 2018 Feb 9.

9. Hussain M, Fizazi K, Saad F, et al. Enzalutamide in men with nonmetastatic, castrationresistant prostate cancer. New Engl J Med 2018;378:2465-74.

10. Smith MR, Cook R, Lee KA, et al. Disease and host characteristics as predictors of time to first bone metastasis and death in men with progressive castration-resistant nonmetastatic prostate cancer. Cancer 2011;117:2077-85.

11. Moreira DM, Howard LE, Sourbeer KN, et al. Predictors of time to metastasis in castration-resistant prostate cancer. Urology 2016;96:171-6. Epub 2016 June 19.

12. Parker CC, James ND, Brawley CD, et al. Radiotherapy to the primary tumour for newly diagnosed, metastatic prostate cancer (STAMPEDE): a randomised controlled phase 3 trial. Lancet 2018;392:2353-66. Epub 2018 Oct 26.

13. Khalef D, Annala M, Finch DL, et al. Phase 2 randomized cross-over trial of abiraterone + prednisone $(\mathrm{ABI}+\mathrm{P})$ vs enzalutamide $(\mathrm{ENZ})$ for patients $(\mathrm{pts})$ with metastatic castration resistant prostate cancer (mCPRC): results for 2nd-line therapy. J Clin Oncol 2018;36 Suppl 15:S5015 Epub 2918 June 1.

14. Miyake H, Hara T, Ozono S, et al. Impact of prior use of an androgen receptor-axistargeted (ARAT) agent with or without subsequent taxane therapy on the efficacy of another ARAT agent in patients with metastatic castration-resistant prostate cancer. Clin Genitourin Canc 2017;15:e217-22.

15. Maughan BL, Boudadi K, Nadal RM, et al. Intercalating docetaxel between novel hormone therapies (NHT) abiraterone and enzalutamide in metastatic castration resistant prostate cancer (mCRPC): does it resensitize patients to the second NHT agent? J Clin Oncol 2017;34 Suppl 15:e16550. Epub 2017 May 11. 
16. Smith MR, Saad F, Rathkopf DE, et al. Clinical outcomes from androgen signalingdirected therapy after treatment with abiraterone acetate and prednisone in patients with metastatic castration-resistant prostate cancer: post hoc analysis of COU-AA-302. Eur Urol 2017;72:10-3. Epub 2017 Mar 21.

17. Hoyle AP, Ali SA, James ND, et al. LBA4 effects of abiraterone acetate plus prednisone/prednisolone in high and low risk metastatic hormone sensitive prostate cancer. Ann Oncol 2018;29 Suppl 8:722. 


\section{Figures and Tables}

\section{Fig. 1.}

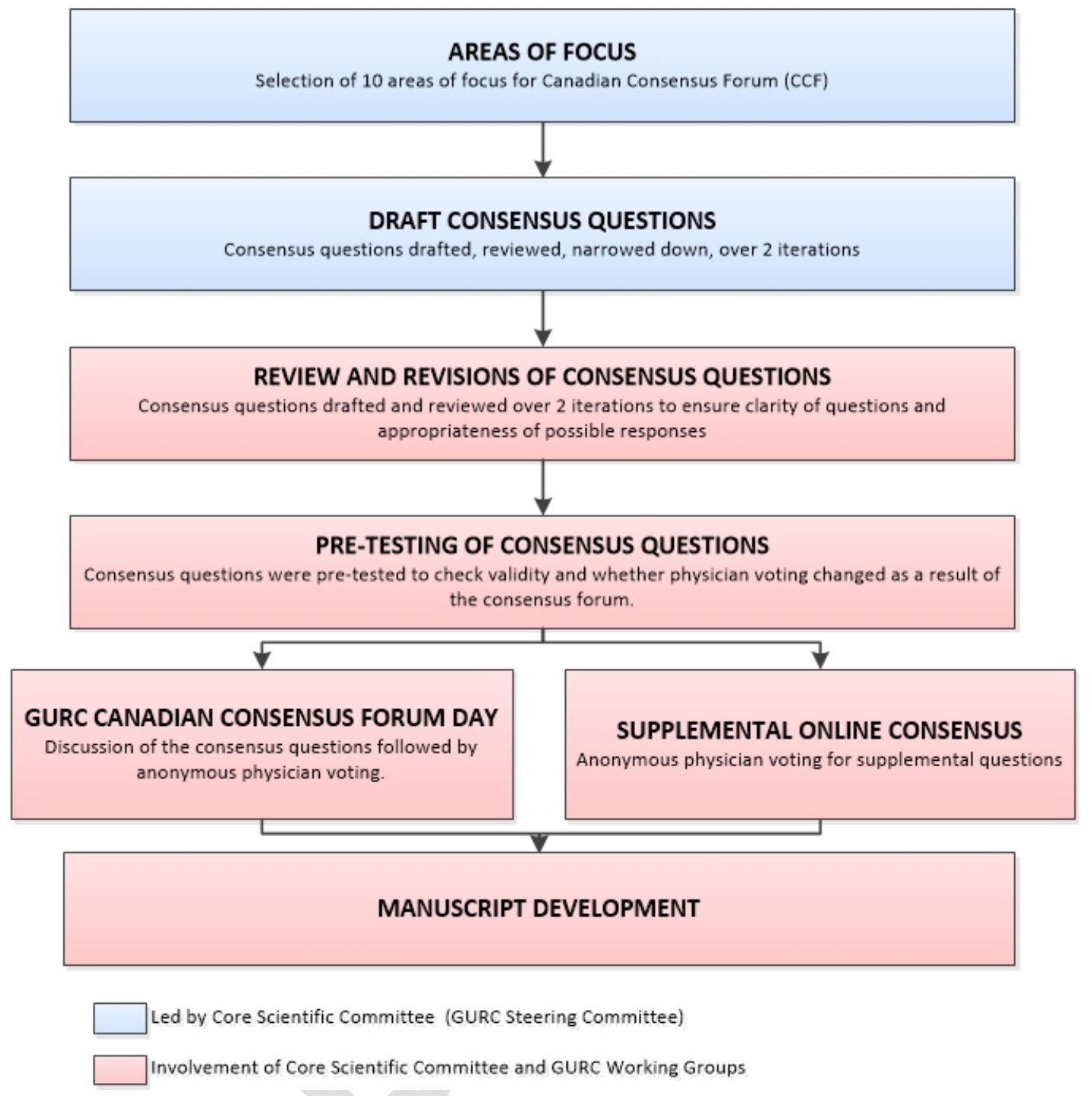


Fig. 2.

\section{Sequencing $\mathrm{nmCRPC} \rightarrow \mathrm{mCRPC}$}

Consensus

Level

$81-85 \%$

Apalutamide or Enzalutamide

Docetaxel

\section{Sequencing $\mathrm{mCSPC} \rightarrow \mathrm{mCRPC}$}

$\begin{gathered}\text { Consensus } \\ \text { Level }\end{gathered}$
$100 \%$

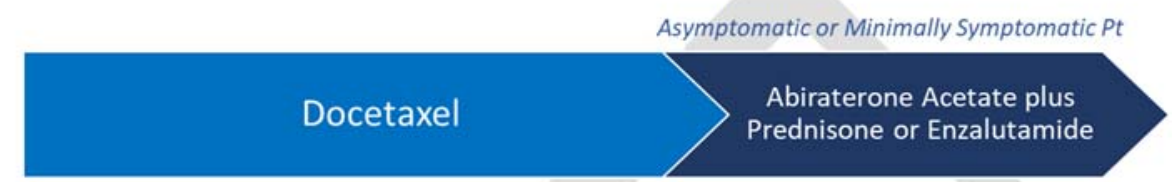

Asymptomatic or Minimally Symptomatic Pt

$78 \%$

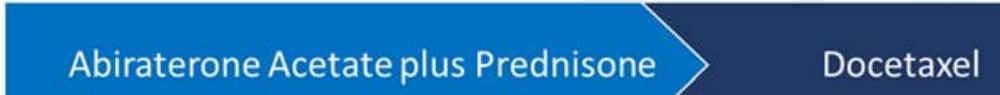

Symptomatic Pt

$96.2 \%$
Docetaxel 


\begin{tabular}{|c|c|}
\hline \multicolumn{2}{|l|}{ Biochemical recurrence } \\
\hline $\begin{array}{l}\text { In general, absolute prostate specific antigen should be used to guide when to initiate } \\
\text { ADT after biochemical recurrence following local radical treatment. }\end{array}$ & $89 \%$ \\
\hline $\begin{array}{l}\text { Intermittent ADT should generally be used for patients with no documented metastatic } \\
\text { disease and PSA-only recurrence following local radical treatment. }\end{array}$ & $92.6 \%$ \\
\hline $\begin{array}{l}\text { On average, PSA should be measured every 3-4 months for PSA recurrence after local } \\
\text { radical therapy. }\end{array}$ & $92.6 \%$ \\
\hline \multicolumn{2}{|l|}{ Non-metastatic castration-resistant prostate cancer } \\
\hline $\begin{array}{l}\text { For the majority of patients, a PSADT of } \leq 10 \text { months should be used as the threshold } \\
\text { to start second-generation AR therapy for patients with nmCRPC. }\end{array}$ & $78 \%$ \\
\hline $\begin{array}{l}\text { For the majority of patients, a PSADT of } \leq 10 \text { months should be used as the threshold } \\
\text { to start second-generation AR therapy for patients with nmCRPC and PSA } 10-20 \\
\mathrm{ng} / \mathrm{mL} \text {. }\end{array}$ & $88.9 \%$ \\
\hline $\begin{array}{l}\text { For patients with non-metastatic CRPC on conventional imaging and PSADT } \leq 10 \\
\text { months, treatment should be initiated with nmCRPC agents such as apalutamide or } \\
\text { enzalutamide. }\end{array}$ & $96.3 \%$ \\
\hline $\begin{array}{l}\text { For the majority of patients with non-metastatic CRPC on conventional imaging, } \\
\text { metastases on PET-based imaging, and PSADT } \leq 10 \text { months, treatment with nmCRPC } \\
\text { agents such as apalutamide or enzalutamide is recommended. }\end{array}$ & $88.9 \%$ \\
\hline $\begin{array}{l}\text { Surrogate endpoints likely correlated with overall survival (OS), such as metastasis- } \\
\text { free survival (MFS), provide sufficient evidence for treatment decision-making in } \\
\text { nmCRPC. }\end{array}$ & $100 \%$ \\
\hline \multicolumn{2}{|l|}{ Metastatic castration-sensitive prostate cancer } \\
\hline $\begin{array}{l}\text { For the majority of men presenting with high-volume mCSPC, ADT treatment in the } \\
\text { form of luteinizing hormone-releasing hormone (LHRH) agonist alone }(+/ \text { - short } \\
\text { course first-generation androgen receptor [AR] antagonist) is recommended. }\end{array}$ & $81.5 \%$ \\
\hline $\begin{array}{l}\text { For the majority of patients with de novo low-volume mCSPC who are not } \\
\text { symptomatic from the primary tumour, treatment of the primary tumour is } \\
\text { recommended in addition to systemic therapy. }\end{array}$ & $74.1 \%$ \\
\hline $\begin{array}{l}\text { For the majority of patients with de novo low-volume mCSPC, radiation the } \\
\text { preferred form of treatment of the primary tumour. }\end{array}$ & $96.3 \%$ \\
\hline
\end{tabular}




\begin{tabular}{|c|c|}
\hline $\begin{array}{l}\text { In men with de novo high-volume mCSPC who are not symptomatic from the primary } \\
\text { tumour, treatment of the primary tumour in addition to systemic therapy is not } \\
\text { recommended. }\end{array}$ & $78 \%$ \\
\hline \multicolumn{2}{|l|}{ Sequencing of treatments across the disease spectrum } \\
\hline $\begin{array}{l}\text { In patients who receive apalutamide for nmCRPC and subsequently progress to } \\
\mathrm{mCRPC} \text {, docetaxel is recommended for first-line treatment of mCRPC (with or without } \\
\text { stereotactic body radiotherapy). }\end{array}$ & $81.5 \%$ \\
\hline $\begin{array}{l}\text { In patients who receive enzalutamide for nmCRPC and subsequently progress to } \\
\mathrm{mCRPC} \text {, docetaxel is recommended for first-line treatment of } \mathrm{mCRPC} \text { (with or without } \\
\text { stereotactic body radiotherapy). }\end{array}$ & $85.2 \%$ \\
\hline $\begin{array}{l}\text { For the majority of asymptomatic or minimally symptomatic men who received } \\
\text { docetaxel in the castration-sensitive setting, abiraterone acetate }+ \text { prednisone or } \\
\text { enzalutamide is the preferred first-line treatment option for mCRPC. }\end{array}$ & $100 \%$ \\
\hline $\begin{array}{l}\text { For the majority of asymptomatic or minimally symptomatic men who received } \\
\text { abiraterone acetate }+ \text { prednisone in the castration-sensitive setting, docetaxel is the } \\
\text { preferred first-line treatment option for mCRPC. }\end{array}$ & $78 \%$ \\
\hline $\begin{array}{l}\text { For the majority of symptomatic men who received abiraterone acetate }+ \text { prednisone in } \\
\text { the castration-sensitive setting, docetaxel is the preferred first-line treatment option for } \\
\text { mCRPC. }\end{array}$ & $96.2 \%$ \\
\hline $\begin{array}{l}\text { For the majority of asymptomatic men who were treated with abiraterone acetate }+ \\
\text { prednisone or enzalutamide for first-line mCRPC and who had an initial response } \\
\text { followed by PSA only progression (secondary acquired resistance), continuation on } \\
\text { current therapy is recommended. }\end{array}$ & $77.8 \%$ \\
\hline $\begin{array}{l}\text { For the majority of asymptomatic men who were treated with abiraterone acetate }+ \\
\text { prednisone or enzalutamide for first-line } \mathrm{MCRPC} \text { and who had initial response } \\
\text { followed by radiologic }+ \text { PSA progression (secondary acquired resistance), docetaxel is } \\
\text { the preferred second-line treatment. }\end{array}$ & $100 \%$ \\
\hline $\begin{array}{l}\text { For the majority of symptomatic men who were treated with abiraterone acetate }+ \\
\text { prednisone or enzalutamide for first-line mCRPC and who had an initial response } \\
\text { followed by progression, docetaxel is the preferred second-line treatment. }\end{array}$ & $96.3 \%$ \\
\hline $\begin{array}{l}\text { For the majority of men with mCRPC who are progressing on or after docetaxel for } \\
\text { mCRPC, abiraterone acetate }+ \text { prednisone, or enzalutamide is the preferred second-line } \\
\text { treatment for men without prior abiraterone acetate }+ \text { prednisone or enzalutamide } \\
\text { treatment. }\end{array}$ & $100 \%$ \\
\hline ptomat & $85.2 \%$ \\
\hline
\end{tabular}




\begin{tabular}{|c|c|}
\hline prednisone, a steroid switch to dexamethasone is reco & \\
\hline Metastatic castration-resistant prostate cancer & \\
\hline $\begin{array}{l}\text { For the majority of asymptomatic or minimally symptomatic men with mCRPC who } \\
\text { did not receive docetaxel or abiraterone acetate }+ \text { prednisone in the castration-sensitive } \\
\text { setting, abiraterone acetate }+ \text { prednisone or enzalutamide is the preferred first-line } \\
\text { treatment for mCRPC. }\end{array}$ & $100 \%$ \\
\hline $\begin{array}{l}\text { Chemotherapy used after initial ARAT therapy is not felt to restore sensitivity to } \\
\text { further ARAT use. }\end{array}$ & $74.1 \%$ \\
\hline $\begin{array}{l}\text { In the mCRPC setting, fatigue related to enzalutamide was treated with a dose } \\
\text { reduction of enzalutamide. }\end{array}$ & $88.9 \%$ \\
\hline Genetic testing & \\
\hline $\begin{array}{l}\text { In men with DNA repair defects (germline or somatic) who progress early on ADT to } \\
\text { mCRPC, first-line mCRPC should be treated with standard options. }\end{array}$ & $78 \%$ \\
\hline $\begin{array}{l}\text { In men with newly diagnosed metastatic (M1) prostate cancer, genetic counselling and } \\
\text { testing is recommended in a minority of selected patients. }\end{array}$ & $74.1 \%$ \\
\hline $\begin{array}{l}\text { In men with newly diagnosed metastatic (M1) prostate cancer, genetic counselling and } \\
\text { testing is recommended for men with a positive family history for prostate } \\
\text { cancer/breast cancer/ovarian cancer. }\end{array}$ & $88.9 \%$ \\
\hline $\begin{array}{l}\text { In men with newly diagnosed metastatic (M1) prostate cancer, genetic counselling and } \\
\text { testing is recommended for men with a positive family history for other cancer } \\
\text { syndromes (e.g. hereditary breast cancer and ovarian cancer syndrome and/or } \\
\text { pancreatic cancer, or Lynch syndrome). }\end{array}$ & $74 \%$ \\
\hline & \\
\hline $\begin{array}{l}\text { For the majority of men with } \mathrm{mCSPC}, \mathrm{CT} \text { and bone scintigraphy is the recommended } \\
\text { imaging modality. }\end{array}$ & $77.8 \%$ \\
\hline $\begin{array}{l}\text { For men with CSPC who have received local treatment with curative intent (+/- salvage } \\
\text { radiation therapy), PET-CT (PSMA, Choline or FACBC (fluciclovine)) imaging is the } \\
\text { modality recommended to diagnose an oligometastatic recurrent state. }\end{array}$ & $74.1 \%$ \\
\hline
\end{tabular}




\section{Appendix}

\section{Non-metastatic castrate-resistant prostate cancer}

\section{Selected areas of $>50 \%$ panel agreement}

When to switch therapy: $37 \%$ of physicians indicated they would wait for radiographic progression before switching therapy to subsequent therapy following apalutamide or enzalutamide. No physicians felt PSA-only progression was sufficient to switch therapy. 59\% of physicians indicated they would wait for another type of progression in addition to radiographic progression.

Asymptomatic oligometastatic progression: 70\% of physicians indicated they would continue treatment and treat metastatic sites (ie stereotactic body radiotherapy) in patients who develop asymptomatic oligmetastatic disease while on apalutamide or enzalutamide, while 19\% would switch treatment to another AR therapy, citing the switch to abiraterone plus prednisone in the SPARTAN study. During the discussion, the Panel clarified that they interpreted progression in this question as the appearance of the first (SINGLE) oligometastatic site. While there was a reasonably high level of agreement on the preferred treatment approach in this question, this was highlighted as an area where further research is needed. Chemotherapy, while not the leading approach here, was noted to still play a role in select patients.

Role of PFS2 data: In terms of the interpretation of PFS2 data, 52\% of physicians indicated that the PFS2 evidence is promising and would like to see overall survival results following final analysis of SPARTAN and 44\% indicated that the data supports early treatment. Collectively, $96 \%$ of physicians felt that the PFS2 evidence is promising or supports that the 
magnitude of benefit seen with initiating treatment earlier in the nmCRPC state is greater than delaying treatment initiation to early mCRPC.

Treatment selection in the absence of contraindications: $70 \%$ of physicians indicated having no preferred choice between apalutamide or enzalutamide in the absence of contraindications and assuming equivalent access to both therapies, while $26 \%$ preferred apalutamide and 4\% preferred enzalutamide. During the discussion, one reason cited for preference for apalutamide was PSF2 results from SPARTAN and anecdotal experience of having to reduce the dose enzalutamide for fatigue in an otherwise asymptomatic patient population. Reasons cited for preference of enzalutamide over apalutamide was familiarity with the agent and experience with its use.

Treatment selection in patients with comorbidities: The presence of patient comorbidities was not observed to definitively influence treatment selection between agents for nmCRPC (Supplementary Fig. 1). Overall, no clear preference between the agents was observed for patients with pre-existing hypertension, renal insufficiency, pre-existing arrhythmias, pre-existing cardiac ejection fracture $<45 \%$, and active liver disease. While not reaching threshold of consensus, greater than half of physicians indicated a preference for apalatumide with ongoing ADT in patients with pre-existing cognitive impairment or significant fatigue, and enzalutamide in patients with pre-existing hypothyroidism.

Managing side effects: There were variable comfort levels in managing side effects related to AR therapy, and differences in referral practices for management of AEs (Supplemntary Fig. 2). Prostate cancer specialists appear to be comfortable managing osteoporosis or osteopenia, rash, and fatigue, but would prefer to refer to specialists for other adverse events. 


\section{Metastatic castration-sensitive prostate cancer}

\section{Selected areas of $>50 \%$ panel agreement}

High volume/high-risk mCSPC: $67 \%$ of physicians indicating they would add abiraterone actate plus prednisone to ADT in this subgroup of mCSPC patients, while $33 \%$ of physicians indicated they would add docetaxel.

Low volume/low-risk mCSPC: $63 \%$ of physicians indicated they would add abiraterone actate plus prednisone to ADT, while $33 \%$ of physicians indicated they would just continue ADT alone. No physicians recommended the use of docetaxel in this patient population. During the discussion, the panel discussed the absolute magnitude of overall survival benefit (4.4\%) reported from STAMPEDE for use of abiraterone acetate plus prednisone in the low volume mCSPC subset, noting that the magnitude of benefit was small but even small benefits of overall survival may be worth considering as a potential treatment option. ${ }^{17}$ The panel also debated the appropriate interpretation of the data, given that this subset analysis was a retrospective post-hoc analysis of the STAMPEDE data.

Treatment selection: The presence of certain prognostic or patient features were observed to guide treatment selection to varying degrees. A preference for the use of abirateroneprednisone was observed for young healthy patients with low volume disease $(89 \%$ of physicians), Gleason $>8$ ( $78 \%$ of physicians), extensive bulky lymph nodes only ( $89 \%$ of physicians), high volume metastatic disease with poor performance ( $85 \%$ of physicians). A preference for the use of docetaxel was observed for young healthy patients with high-volume disease (74\% of physicians), high volume metastases with low PSA (78\% of physicians) (Supplementary Fig. 3) 


\section{Metastatic castration-resistant prostate cancer}

\section{Selected areas of $>50 \%$ panel agreement}

Dose reductions for treatment side effects: When using abiraterone acetate plus prednisone, 55\% of physicians indicated they would attempt dose reduction of abiraterone acetate in a majority of their patients for side effects other than elevated liver function enzymes. For enzalutamide, 70\% of physicians indicated they would attempt a dose reduction of enzalutamide in a majority of their patients. If the side effect was fatigue, $89 \%$ of physicians indicated they attempt a dose reduction of enzalutamide. During the discussion, the panel felt it was important to further elaborate that in their anecdotal experience, the proportion of patients experiencing a significant adverse event that would require dose reduction or a change in management, is generally lower with abiraterone acetate plus prednisone compared to enzalutamide.

Treatment selection: Certain co-morbidities were observed to influence treatment selection to varying degrees. A preference for abiraterone plus prednisone was observed among patients with a history of falls ( $74 \%$ of physicians), baseline fatigue ( $96 \%$ of physicians), and baseline neurocognitive impairment ( $83 \%$ of physicians). A preference for enzalutamide was observed among patients with diabetes ( $82 \%$ of physicians), (Supplementary Fig. 4) 


\section{Oligometastatic prostate cancer}

\section{Selected areas of $>50 \%$ panel agreement}

Definition of oligometastatic prostate cancer: $70 \%$ of physicians defined this as a limited number of bone and/or lymph node metastases that can be treated with local therapy, and $26 \%$ of physicians defined it as a limited number of any metastases (including visceral). During the discussion, the panel noted this as an area where further study is needed.

Cutoff number of metastases for oligometastatic prostate cancer: $59 \%$ of physicians felt the appropriate cut-off was $\leq 5$ metastases while $33 \%$ of physicians felt the appropriate cut-off was $\leq 3$ metastases.

\section{Selected areas of variability in patient management}

Variability in physician voting was seen in the treatment approach for patients with oligometastatic castration-sensitive prostate cancer, and role of metastasis-directed therapy.

Variability in physician voting was seen in the treatment with newly-diagnosed oligometastatic castration-sensitive prostate cancer with an untreated primary tumour, where $48 \%$ of physicians would treat with ADT +/- docetaxel or abiraterone acetate plus prednisone, $26 \%$ would give radical therapy to all lesions including primary lesion + ADT 24-36 mo +/- docetaxel (if high volume) or abiraterone plus prednisone, and $26 \%$ would do the above, but give lifelong ADT. During the discussion, the panel expressed the need for clinical trials to study this scenario. Variability in physician voting was also seen in the treatment of men with recurrent castrationsensitive oligometastatic prostate cancer after receiving local treatment with curative intent $(+/-$ salvage radiation therapy), where $48 \%$ would treat with lifelong ADT +/- docetaxel +/- 
abiraterone acetate plus prednisone, $26 \%$ would add radical metastasis-directed therapy (surgery or RT) to the above, 15\% would treat with metastasis-directed therapy with ADT 24-36 months, and $7 \%$ would treat with metastasis-directed therapy only.

If considering metastasis-directed therapy for men with recurrent castration-sensitive oligometastatic prostate cancer limited to pelvic lymph node metastases (detected by conventional imaging) in pelvis after curative intent local treatment with curative intent $(+/$ - salvage radiation therapy), $30 \%$ of physician would treat with ADT only, $26 \%$ would whole pelvis radiotherapy $+/-$ boost to the suspicious nodes, $22 \%$ would give stereotactic body radiation therapy (SBRT), and $19 \%$ would do a salvage lymph node dissection.

\section{Access to treatments}

\section{Public reimbursement for coverage of using a second AR agent in $\mathrm{MCRPC}$}

When physicians voted on whether provincial funding should be available for use of a secondgeneration $\mathrm{AR}$ agents in patients with $\mathrm{mCRPC}, 48 \%$ of physicians indicated that public provincial reimbursement should be provided for a second AR agent post-docetaxel (sandwich use), and $33 \%$ indicated yes, with no limitations irrespective of sandwich use. Collectively, this suggests that $81 \%$ of physicians see value in access to a $2^{\text {nd }}$ AR agent. During the discussion, the panel elaborated on the value of making funding available so that physicians can have the flexibility to apply their expertise to choose the best treatment course of the patient at this stage. 


\section{Public reimbursement for coverage of a second AR agent after use in mCSPC or nmCRPC}

$52 \%$ indicated yes, with no limitations and $41 \%$ indicated yes, to be used with chemotherapy in between. Collectively, this suggests that $93 \%$ of physicians see value in access to a $2^{\text {nd }} A R$ agent.

\section{Genetic testing and counselling}

No supplemental content.

\section{Referrals}

\section{Selected areas of $>50 \%$ panel agreement}

Referral of patients with nmCRPC: $67 \%$ of the panel felt that referral to the local physician (any specialty) who is most familiar with the management of these patients was appropriate, $26 \%$ felt that referral should be made to a regional cancer centre for opinion/management by multidisciplinary group.

Referral of patients with mCSPC: $52 \%$ of the panel felt patients should be referred to a regional cancer centre for opinion/management by multidisciplinary group, $33 \%$ felt patients should be referred to a local physician (any specialty) who is most familiar with management of these patients. During the discussion, it was highlighted that the management of mCSPC is more complex than the nmCRPC patients.

Referral of patients with mCRPC: $41 \%$ of the panel felt patients should be referred to a regional cancer centre for opinion/management by multidisciplinary group, and $41 \%$ felt patients should be referred to a local physician (any specialty) who is most familiar with management of these patients. 
Overall, these results demonstrate that $>80 \%$ of the panel recommend referral of these patients to experienced clinicians or centres specialized in treating advanced prostate cancer.

\section{Genetic testing and counselling}

\section{Selected areas of variability in patient management}

Variability in practice was seen in the panel's perspectives on the role of PARP inhibitors for mCRPC, treatment plan for low-risk clinically localized prostate cancer in the presence of germline mutations, and newly diagnosed metastatic hormone sensitive prostate cancer in the presence of DNA repair defects.

PARP inhibitor for mCRPC in the presence of DNA repair defects (germline or somatic): $44 \%$ of physicians would use a PARP inhibitor in the majority of their patients if available, and $30 \%$ were not sure. During the discussion, the panel highlighted that this is still an experimental treatment option in Canada and requires more research.

Low-risk clinically localized prostate cancer and germline BRCA1, BRCA2, or ATM mutation: $63 \%$ of physicians indicated that the presence of germline BRCA1, BRCA2, or ATM mutation would make them less likely to offer surveillance, while $33 \%$ would not recommend active surveillance.

Newly diagnosed metastatic hormone sensitive prostate cancer in the presence of DNA repair defects (germline or somatic): $59 \%$ of physicians indicated they would continue with standard treatment while $37 \%$ would be more likely to recommend addition of docetaxel to ADT. 


\section{Imaging}

\section{Selected areas of $>50 \%$ panel agreement}

Frequency of imaging in mCPSC: $65 \%$ of the panel indicated they would image at baseline and then at PSA nadir and progression (confirmed PSA rise or clinical progression).

Frequency of imaging in mCRPC: $56 \%$ would image at baseline and then at PSA nadir and progression (confirmed PSA rise or clinical progression) and 30\% would image at baseline then regular imaging every 3 to 6 months.

The voting results generally reflected that whether patients were $\mathrm{mCSPC}$ or $\mathrm{mCRPC}$, that the majority of the panel would image at baseline, nadir, then at progression while a small proportion would image regularly every 3-6 months 


\section{Supplementary Figures and Tables}

\section{Supplementary Fig. 1.}

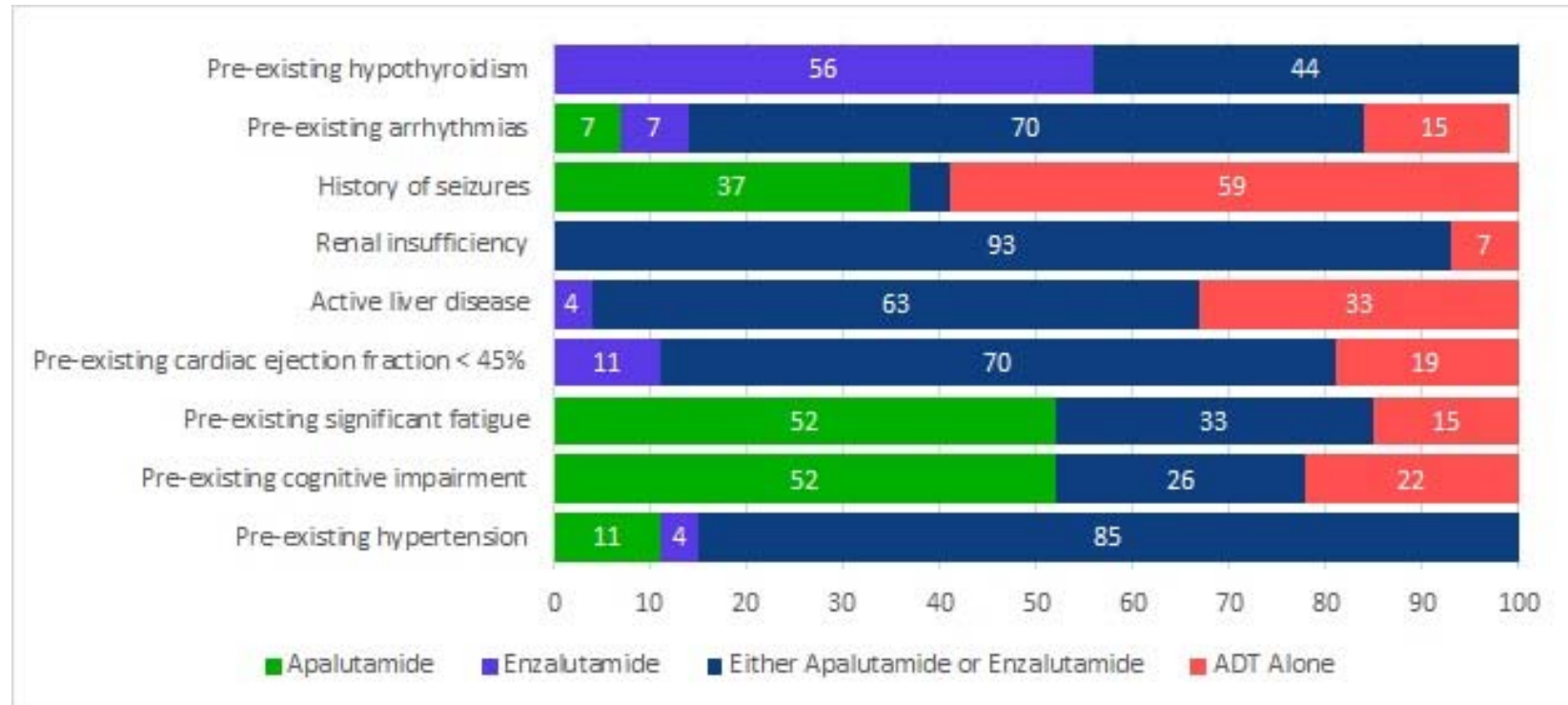

Supplementary Fig. 2.

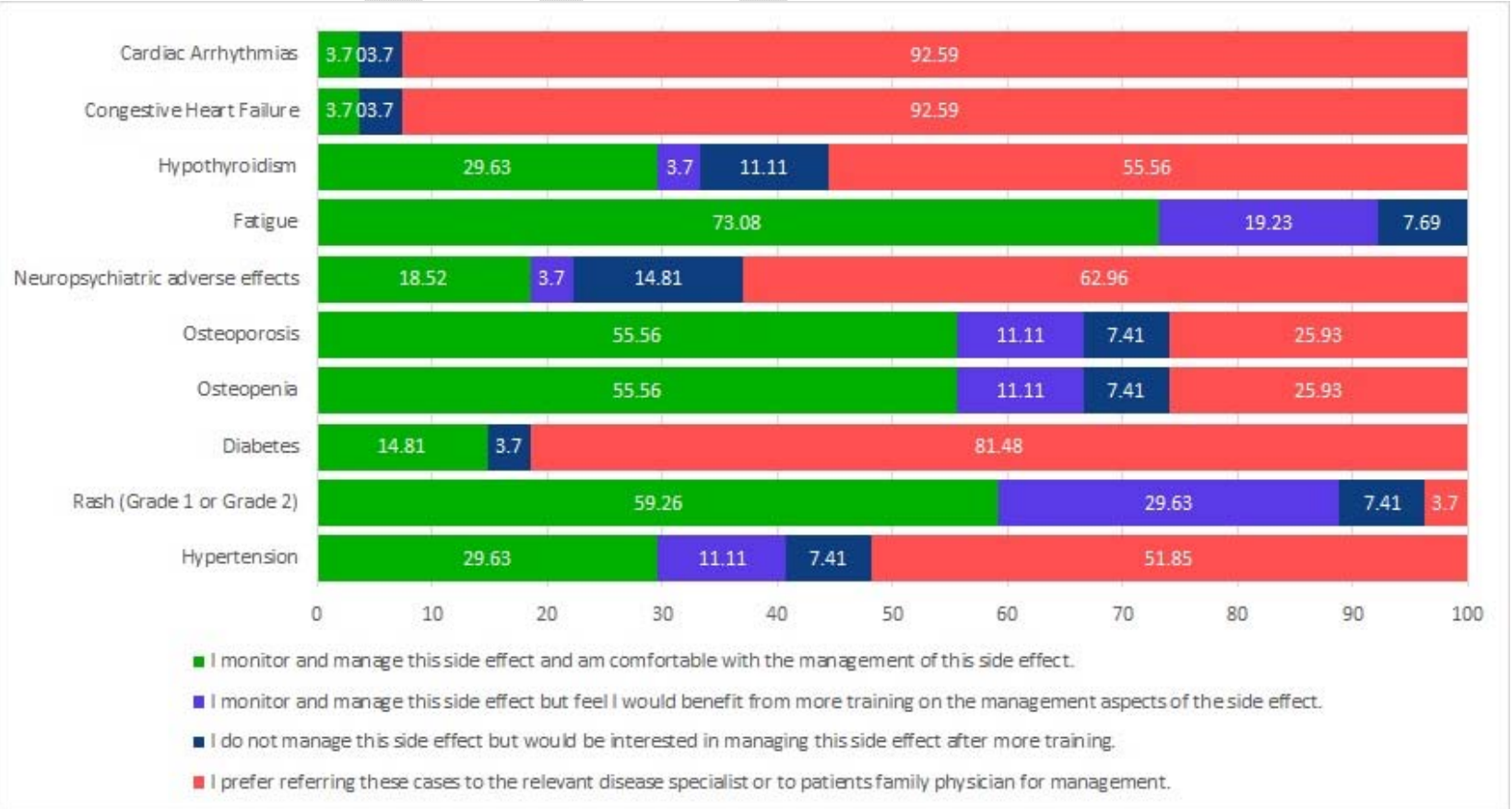




\section{Supplementary Fig. 3.}

High-volume metastases with elevated PSA

High-volume metastases with low PSA

High metastatic burden + poor performance status Both visceral and bone metastases Visceral and lymph node dise ase Extensive bulky lymph nodes only Gleason $>8$

Younger healthy patients with good performance status - Low Volume disease

Younger healthy patients with good performance status - High volume dise ase

Poor performance status (e.g., frail eiderly etc.)

- Docetaxel Abiraterone acetate ADT Alone

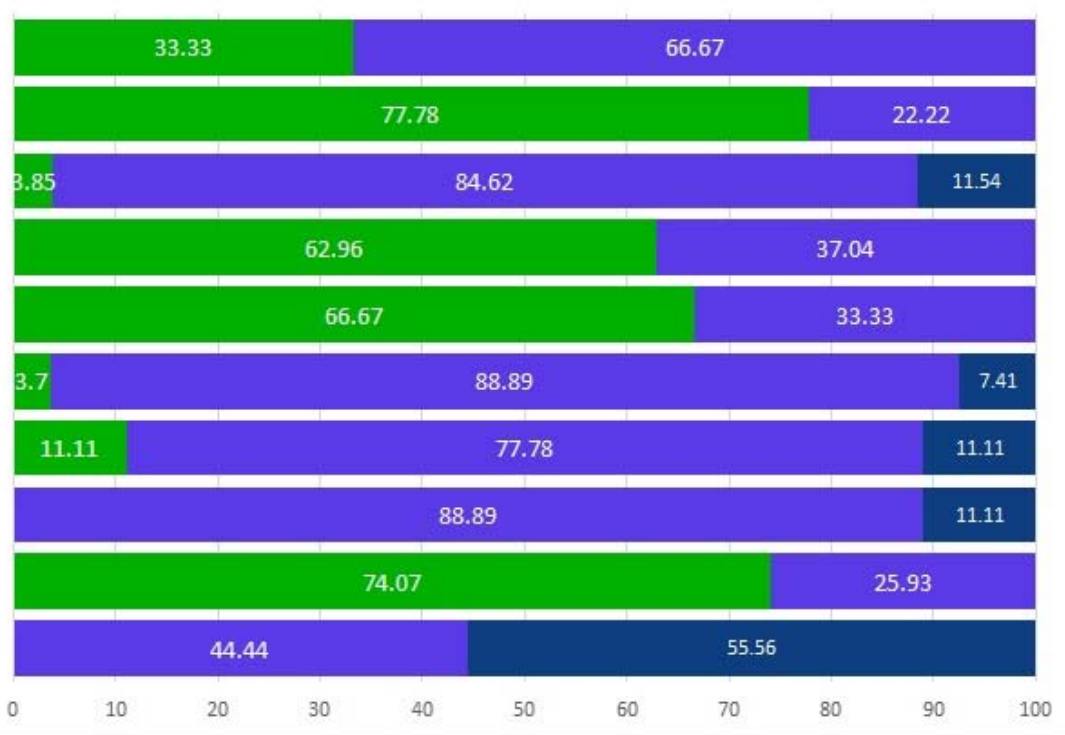

\section{Supplementary Fig. 4.}

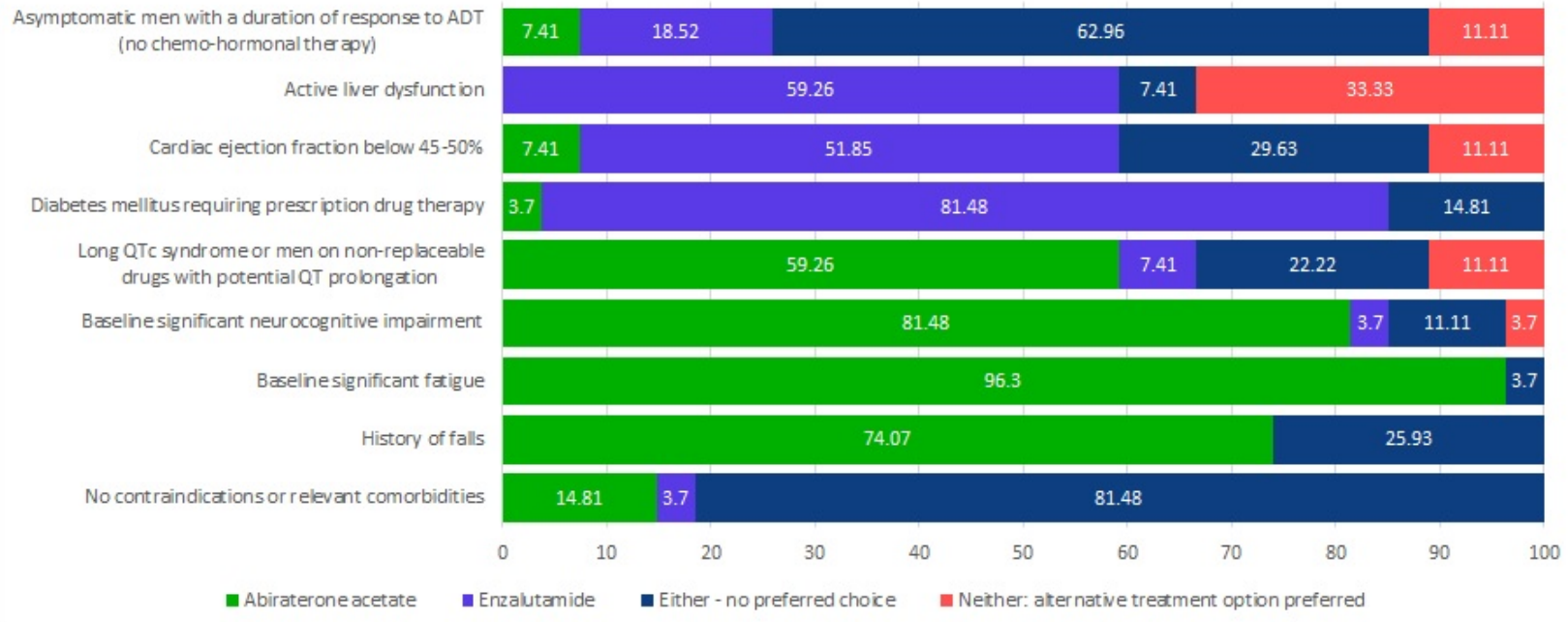




\begin{tabular}{|l|l|l|l|}
\hline \multicolumn{4}{|l}{ Supplementary Table 1. Canadian consensus forum members by specialty and region } \\
\hline First name & Last name & Region & Specialty \\
\hline Fred & Saad & QC & Urologist \\
\hline Kim & Chi & BC & Medical oncologist \\
\hline Tony & Finelli & ON & Urologist \\
\hline Sebastien & Hotte & ON & Medical oncologist \\
\hline Christina & Canil & ON & Medical oncologist \\
\hline Alan & So & BC & Urologist \\
\hline Shawn & Malone & ON & Radiation Oncologist \\
\hline Bobby & Shayegan & ON & Urologist \\
\hline Lorne & Aaron & QC & Urologist \\
\hline Naveen & Basappa & AB & Medical oncologist \\
\hline Henry & Conter & ON & Medical oncologist \\
\hline Brita & Danielson & AB & Radiation oncologist \\
\hline Geoffrey & Gotto & AB & Urologist \\
\hline Robert & Hamilton & ON & Urologist \\
\hline Jason & Izard & ON & Urologist \\
\hline Anil & Kapoor & ON & Urologist \\
\hline Michael & Kolinsky & AB & Medical oncologist \\
\hline Aly-Khan & Lalani & ON & Medical oncologist \\
\hline Jean-Baptiste & Lattouf & QC & Urologist \\
\hline Chris & Morash & ON & Urologist \\
\hline Scott & Morgan & ON & Radiation oncologist \\
\hline Tamim & Niazi & QC & Radiation oncologist \\
\hline Krista & Noonan & BC & Medical oncologist \\
\hline Michael & Ong & ON & Medical oncologist \\
\hline Ricardo & Rendon & NS & Urologist \\
\hline Sandeep & Sehdev & ON & Medical oncologist \\
\hline Jeffrey & Spodek & ON & Urologist \\
\hline
\end{tabular}




\begin{tabular}{|c|c|c|c|}
\hline \multicolumn{4}{|c|}{ Supplementary Table 2. Consensus a cross the topic areas } \\
\hline Topics & Questions & Consensus & $\begin{array}{c}\text { No } \\
\text { consensus }\end{array}$ \\
\hline $\begin{array}{l}\text { 1. Biochemical recurrence } \\
\text { following radical local } \\
\text { definitive therapy }\end{array}$ & $4,5,6,(7$ eliminated $)$ & 3 & 0 \\
\hline $\begin{array}{l}\text { 2. Non-metastatic castration- } \\
\text { resistant prostate cancer } \\
\text { (nmCRPC) }\end{array}$ & $\begin{array}{c}8,9,10,11,12,13,14,15,16, \\
19,20,21\end{array}$ & 5 & 7 \\
\hline $\begin{array}{l}\text { 3. Metastatic castration-sensitive } \\
\text { prostate cancer (mCSPC) }\end{array}$ & $\begin{array}{c}22,22 b, 23,24,25,26,27,28 \\
29,30,31\end{array}$ & 4 & 7 \\
\hline $\begin{array}{l}\text { 4. Sequencing of Systemic } \\
\text { Treatment }\end{array}$ & $\begin{array}{c}17,18,32,34,35,36,38,39 \\
40,41,42\end{array}$ & 10 & 1 \\
\hline $\begin{array}{l}\text { 5. Metastatic castration-resistant } \\
\text { prostate cancer (mCRPC) }\end{array}$ & $33,37,43,44,45$ & 3 & 2 \\
\hline 6. Oligometastatic prostate cancer & $46,47,48,49,50$ & 0 & 5 \\
\hline 7. Funding of treatments & 51,52 & 0 & 2 \\
\hline 8. Referral for care & $60,61,62$ & 0 & 3 \\
\hline $\begin{array}{l}\text { 9. Genetic testing in prostate } \\
\text { cancer }\end{array}$ & $53,54,55,56,57,58,59$ & 3 & 4 \\
\hline $\begin{array}{l}\text { 10. Imaging in advanced prostate } \\
\text { cancer }\end{array}$ & $63,64,65,66,67$ & 2 & 3 \\
\hline
\end{tabular}

